\title{
A dose-finding study of miltefosine (hexadecylphosphocholine) in patients with metastatic solid tumours
}

\author{
Jaap Verweij, André Planting, Maria van der Burg, and Gerrit Stoter \\ Department of Medical Oncology, Rotterdam Cancer Institute/Daniel den Hoed Kliniek, \\ Groene Hilledijk 301, 3075 EA Rotterdam, The Netherlands
}

Received 19 February 1992/Accepted 22 April 1992

\begin{abstract}
Summary. The ether lipid miltefosine (hexadecylphosphocholine) was orally given to patients with various tumours in a dose-finding study. All patients initially received a daily total dose of $100 \mathrm{mg}$, which in the absence of side-effects was increased to $150 \mathrm{mg}$ and further to $200 \mathrm{mg}$. A total of 54 patients were entered and were evaluable for gastrointestinal toxicity. Nausea and vomiting were found to be dose-limiting; $22 \%$ of patients ultimately tolerated a dose of $100 \mathrm{mg}, 59 \%$ tolerated a dose of $150 \mathrm{mg}$ and $19 \%$ tolerated a dose of $200 \mathrm{mg}$. In addition $30 \%$ of patients developed renal dysfunction, which was thought to be related to the drug. No other toxities were observed. For further phase II studies it is recommended that one starts with a dose of $150 \mathrm{mg}$ daily, divided over three administrations.
\end{abstract}

Key words: Miltefosine - Hexadecylphosphocholine Dose finding - Solid tumours

\section{Introduction}

Ether lipids have been the subject of laboratory anticancer research for several years. Only recently some of these drugs were introduced in clinical trials, still mainly restricted to phase I studies.

Miltefosine (hexadecylphosphocholine) was one of the first ether lipids to enter clinical studies (Unger et al. $1989 \mathrm{a}, 1990$ ). The drug is a synthetic phospholipid derivative with remote similarity to the major constituent of the cell membrane, lecithin. Therefore, although the detailed mode of action is still unknown, selective interference with membrane functions is presumed.

In vitro the drug is active in the L1210, HL-60, U937 Raji and K562 leukaemia cell lines (Unger et al. 1989 b). Activity was also found in the KB cell line (Fleer et al.

Correspondence to: J. Verweij
1987). Owing to haemolytic effects an i.v. formulation was difficult; however, an oral formulation became available. By oral application the drug was active in vivo in dimethylbenz $[a]$ anthracene-induced breast cancers in mice (Unger et al. 1989 b; Eibl et al. 1988) and mammary human tumour xenografts (Scherf et al. 1987), and had a high bioavailability (Unger et al. 1989 b).

Distribution studies showed a high accumulation in the kidneys, lung and liver (Unger et al. 1989 b; Breiser et al. 1987). In a phase I study in 22 patients the dose-limiting toxicities of miltefosine were nausea and vomiting, which could be reduced by splitting the daily dose into multiple administrations. No other toxicities were noted. The dose recommended for phase II studies was $150 \mathrm{mg} /$ day. However, because the phase I data were scanty, it was decided that a dose-finding study within a large phase II study programme should be performed.

\section{Materials and methods}

Patients with metastatic histologically confirmed colorectal, nonsmall-cell lung or squamous-cell head and neck cancer were eligible for the (dose-finding) study. For colorectal and head and neck cancer one prior form of chemotherapy was permitted. Prior chemotherapy was not allowed for patients with non-small-cell lung cancer. Patients had to have a WHO performance score of 2 or less and normal serum creatinine and bilirubin. There were no age limits. Verbal informed consent was required.

Miltefosine (hexadecylphosphocholine, D 18506) was supplied by ASTA Medica AG in capsules containing $50 \mathrm{mg}$ active drug/ capsule. Treatment consisted of miltefosine $50 \mathrm{mg}$ orally twice daily after meals in the first week. In the absence of side-effects this dose was increased to three times daily in the second week. In the case of a total absence of side-effects a further escalation to four times daily was performed in week 3 or 4 . In the case of nausea and/or vomiting, adequate oral antiemetics were given; if these were ineffective a dose reduction by one step was allowed. Patients not tolerating $50 \mathrm{mg}$ twice daily were taken off study. The highest tolerated dose per patient was given until tumour progression.

Prior to treatment, baseline haematology and chemistry data were obtained. For the first 8 weeks, weekly haemoglobin, white blood cells and platelets were determined; thereafter these were recorded every 4 weeks. Every 4 weeks a complete check of serum 
chemistry, including serum creatinine, sodium, potassium, calcium, bilirubin, alkaline phosphatase and aspartate and alanine aminotransferases, was performed.

Response and toxicity were evaluated according to WHO criteria. For nausea/vomiting the worst grade during the week was recorded, so if a patient vomited once during a week this was considered to be grade 2 .

\section{Results}

A total of 54 patients entered the study, 34 men and 20 women, with a median age of 58 years (range 32-79) and a median WHO performance score of 1 (range 0-2). 31 patients had colorectal cancer, 13 had non-small-cell lung cancer and 10 had squamous-cell head and neck cancer. Seven patients had had prior chemotherapy consisting of 5-fluorouracil ( 3 patients), methotrexate ( 3 patients) and/ or cisplatin (1 patient), or prior immunotherapy with interleukin-2 (2 patients).

A total of 315 weeks of treatment with miltefosine were evaluated. The median duration of treatment was 6 weeks (range 2-8+ weeks). All patients started at a daily dose of $100 \mathrm{mg}$ miltefosine and in $51(94 \%)$ the dose could be increased to $150 \mathrm{mg}$. A further dose escalation to $200 \mathrm{mg}$ daily was performed in 11 patients $(20 \%)$. Nausea and/or vomiting were found to be dose-limiting and occurred in $48 \%$ of the treatment weeks; $29 \%$ grade 1 (WHO) and $19 \%$ grade 2 .

The percentage of nausea/vomiting analysed per dose level over the total number of treatment weeks is given in Table 1.

The dose tolerated until the end of treatment was $100 \mathrm{mg}$ in 12 patients $(22 \%), 150 \mathrm{mg}$ in 32 patients $(59 \%)$ and $200 \mathrm{mg}$ in 10 patients $(19 \%)$. In most patients the worst grade of nausea/vomiting per dose level was observed in the first week of treatment at that dose level. However, in some patients nausea/vomiting did not occur until some weeks after treatment had begun.

Most standard antiemetics (including $5 \mathrm{HT}_{3}$ antagonists) were ineffective in preventing nausea/vomiting. The least emetogenic effect was obtained if miltefosine was taken immediately after meals, with domperidon taken $0.5 \mathrm{~h}$ before the meals at a dose of $20 \mathrm{mg}$.

The only other side-effect observed was renal toxicity. Eight patients could not be evaluated in this respect because there was no follow-up creatinine determination. Renal toxicity consisted of an increase in serum creatinine level and occurred in $14(30 \%)$ out of 46 evaluable patients. In 12 patients it was grade $1(26 \%)$ and in 2 it was grade $2(4 \%)$. In 3 patients treatment had to be stopped because of the renal side-effects; in 2, serum creatinine normalized after the drug was discontinued. In one patient we were unable to observe recovery from this side-effect because in the meantime this patient died from pneumonia. At autopsy, renal changes consisted of interruptions of the epithelial lining of the descending tubules caused by plasmacellular infiltrates.

In 3 patients treatment was stopped because of disease progression at the time that renal toxicity was found. In these patients renal function fully recovered. In 2 of the other remaining 6 patients renal dysfunction normalized
Table 1. Percentage nausea/vomiting per dose level over the total length of treatment

\begin{tabular}{llllr}
\hline $\begin{array}{l}\text { Daily dose } \\
\text { (mg) }\end{array}$ & $\begin{array}{l}\text { Total length of } \\
\text { treatment } \\
\text { (weeks) }\end{array}$ & \multicolumn{2}{l}{$\begin{array}{l}\text { Patients experiencing (\%) } \\
\text { to WHO level: }\end{array}$} \\
\cline { 3 - 5 } & & 0 & 1 & 2 \\
\hline 100 & 94 & 59 & 34 & 7 \\
150 & 188 & 46 & 27 & 27 \\
200 & 33 & 61 & 27 & 12 \\
\hline
\end{tabular}

notwithstanding the continuation of full-dose treatment. In the other 4 patients serum creatinine levels reached a plateau during miltefosine therapy and returned to normal after cessation. The time to recovery from renal toxicity varied from 2 to 30 weeks (median 4). Five patients had not experienced nausea/vomiting at all when renal toxicity occurred; 6 patients experienced nausea and 3 had vomited sporadically. The latter 9 patients denied inadequate fluid intake in the weeks preceding the occurrence of renal toxicity.

There was no myelosuppression, no hair loss and no other sign of organ toxicity.

\section{Discussion}

Our dose-finding study confirmed by and large the doselimiting gastrointestinal toxicity observed in the previous phase I studies (Unger et al. 1990). Nausea and vomiting appear to be dose-dependent as far as the final tolerance of patients is concerned. However, our data indicate that a patient-guided dose escalation is feasible without the frequency of this side-effect being increased.

In addition we report the development of reversible renal dysfunction in $30 \%$ of patients, which is probably caused by an intrinsic renal lesion as demonstrated in one autopsy case. The percentage of renal toxicity may be somewhat overestimated because of the possible effect of a coinciding decrease in the effective circulating volume related to inadequate fluid intake resulting from nausea/ vomiting.

On the basis of animal data, renal toxicity was not an unexpected finding. Although renal toxicity itself was not seen in these studies (Unger et al. 1989 b; Eibl et al. 1988), a high accumulation of the drug in the kidneys was reported (Unger et al. $1989 \mathrm{~b}$; Breiser et al. 1987). As this side-effect was first seen after a median of 5 weeks of treatment (range 3-8), it is difficult to deduce a possible relationship to the daily dose. Although it cannot be completely excluded, there was no obvious relationship to cumulative dose. We did not find any relationship between the renal toxicity and other clinical parameters.

Oral miltefosine completely lacked any other toxicity. As was reported earlier, we only observed two tumour regressions in colorectal cancer patients (Planting et al. 1990). Oral miltefosine can be given safely to patients at a dose of $150 \mathrm{mg} /$ day, divided over three doses. Prophylactic use of oral domperidon is recommended. Careful monitoring of serum creatinine is also advisable. 


\section{References}

Breiser A, Kim DJ, Fleer EAM, Damenz W, Drube A, Berger M, Nagel GA, Eibl H, Unger C (1987) Distribution and metabolism of hexadecylphosphocholine in mice. Lipids 22:925-926

Eibl $\mathrm{H}$, Unger C, Berger M (1988) Characterization of the antitumor activity of hexadecylphosphocholine (D:8506). Eur J Cancer Clin Oncol 24:1457-1461

Fleer EAM, Unger C, Kim DJ, Eibl H (1987) Metabolism of ether phospholipids and analogs in neoplastic cells. Lipids 22:856861

Planting A, Verweij J, Hoff A, Dees A, Seynaeve C, Stoter G (1990) Phase II study of daily oral hexadecylphosphocholine in advanced colorectal cancer. Ann Oncol 1 [Suppl]:46
Scherf HR, Schuler B, Berger MR, Schmähl D (1987) Therapeutic activity of ET-18- $\mathrm{OCH}_{3}$ and hexadecylphosphocholine against mammary tumors in BD-VI rats. Lipids 22:927-929

Unger C, Eibl H, Nagel GA, Heyden $\mathrm{HW}$ von, Breiser A, Engel J, Stekar J, Peukert M, Hilgard P, Berger M (1989a) Hexadecylphosphocholine in the topical treatment of skin metastases: a phase I trial. Contrib Oncol 37:219-223

Unger C, Damenz W, Fleer EAM, Kim DJ, Breiser A, Hilgard P, Engel J, Magel A, Eibl H (1989 b) Hexadecylphosphocholine, a new ether lipid analogue. Studies on the antineoplastic activity in vitro and in vivo. Acta Oncol 28:213-217

Unger C, Eibl HJ, Heyden HW von, Peukert M, Sindermann H, Nagel G (1990) Phase I study with daily hexadecylphosphocholine in patients with malignant disease (abstract). J Cancer Res Clin Oncol 116:993 